\title{
SOLAR NUCLEAR ENERGY GENERATION AND THE CHLORINE SOLAR NEUTRINO EXPERIMENT
}

\author{
H.J. HAUBOLD \\ UN Outer Space Office, Vienna International Centre, Vienna, Austria \\ and \\ A.M MATHAI \\ Department of Mathematics and Statistics, McGill University, Montreal, \\ Canada
}

\begin{abstract}
The study of solar neutrinos may provide important insights into the physics of the central region of the Sun. Four solar neutrino experiments have confirmed the solar neutrino problem but do not clearly indicate whether solar physics, nuclear physics, or neutrino physics have to be improved to solve it. Nonlinear relations among the different neutrino fluxes are imposed by two coupled systems of differential equations governing the internal structure and time evolution of the Sun. We assume that the results of the four neutrino experiments are correct and are concerned not with the discrepancy between the average rate and the predicted rate, but with a possible time dependence of the argon production rate as revealed in the Homestake experiment over a time period of 20 years. Based on the subtlety of the solar neutrino problem we review here qualitatively the physical laws employed for understanding the internal solar structure and conjecture that the interlink between specific nuclear reactions of the PPIII-branch of the proton-proton chain may allow the high-energy solar neutrino flux to vary over time.
\end{abstract}




\section{Solar Neutrino Detection: Results}

The Sun is supposed to be a simple main-sequence star that generates its energy through the proton-proton chain and to a much less extend through the CNO cycle, thereby producing a copious flux of neutrinos. The Sun is considered to be a simple star because very basic physical laws can be employed to describe the evolution and the internal structure of the Sun, namely Newton's laws of gravity and motion, the first two laws of thermodynamics, Einstein's law of equivalence of mass and energy, Boyle's law and Charles' law of perfect gases, and Heisenberg's uncertainty principle. To probe the simplicity and consistency of theoretically developed standard solar models, over the past two and a half decades four experiments to detect the solar neutrino flux have been established (Bahcall and Pinsonneault 1992).

(1) The chlorine experiment in the Homestake gold mine (USA), in operation since 1967, observed an average ${ }^{37}$ Ar production rate by solar neutrinos with energies $E_{\nu}>0.8 \mathrm{MeV}$ of $(2.2 \pm 0.2)$ SNU円 for runs 18-109 over the time period 1970.8 to 1990.0 (Davis 1993).

(2) The neutrino-electron scattering experiment in the Kamiokande mine (Japan), in operation since 1986 for two time periods of 1040 days (Kamiokande II: Jan87-Apr90, 450 days with $E_{\nu}>9.3 \mathrm{MeV}$ and 590 days with $E_{\nu}>7.5$ $\mathrm{MeV}$ ) and 220 days (Kamiokande III: Dec90-Dec91, $E_{\nu}>7.5 \mathrm{MeV}$ ), detected solar neutrinos with event rates $\left\langle\Phi\left({ }^{8} B\right)_{o b s}\right\rangle=[0.47 \pm 0.05$ (stat $) \pm$ $0.06($ syst $)] \Phi_{\text {calc }}\left({ }^{8} B\right)$ of that predicted by standard solar models (Nakamura 1993).

For the chlorine experiment the standard solar model with the best input parameters predicts an event rate of $(8.0 \pm 3.0)$ SNU (Bahcall and Pinsonneault 1992). The experimental results referred to in (1) and (2) above revealed that the measured solar neutrino fluxes are significantly below those predicted by standard solar models, known as the "solar neutrino problem". (3) The gallium experiment under a mountain in the North Caucasus at Baksan (Russia) began to detect solar neutrinos with energies $E_{\nu}>0.23 \mathrm{MeV}$ in 1988 and reported a ${ }^{71} \mathrm{Ge}$ production rate of $\left(85_{-32}^{+22}[\right.$ stat $] \pm 20[$ syst $\left.]\right) \mathrm{SNU}$ (Anosov et al. 1993).

(4) The European gallium experiment in the Gran Sasso tunnel (Italy), in operation since 1991, measured a capture rate of solar neutrinos by ${ }^{71} \mathrm{Ga}$

\footnotetext{
${ }^{1} 1 \mathrm{SNU}$ (solar neutrino unit) $=10^{-36}$ captures per atom per second
} 
of $(79 \pm 10[$ stat $] \pm 6[$ syst $])$ SNU in 30 runs between May 1991 (GALLEX I: May91-March92) and October 1993 (GALLEX II: Aug92-Oct93)(Anselmann et al. 1994).

The detected solar neutrino fluxes for the two gallium experiments almost agree with each other but are in conflict with standard solar models which predict a capture rate of $\left(132_{-17}^{+21}\right)$ SNU (Bahcall and Pinsonneault 1992).

The result from the neutrino-electron scattering experiment (Kamiokande) constrains only the high-energy ${ }^{8} B$ neutrino flux, while the chlorine and gallium experiments (Homestake, Baksan, Gran Sasso) constrain both the ${ }^{7} \mathrm{Be}$ and ${ }^{8} B$ solar neutrino fluxes. The results of the four neutrino experiments, despite of extensive experimental and theoretical efforts to reveal the origin of the discrepancies, do not indicate clearly whether solar physics, nuclear physics, or neutrino physics have to be improved to settle the account of the solar neutrino problem.

The net reaction for the proton-proton chain and the CNO cycle is the conversion of hydrogen into helium,

$$
4 p \longrightarrow \alpha+2 \nu_{e}+2 e^{+}+Q, Q=26.7 \mathrm{MeV},
$$

where two neutrinos are produced in the Sun per 26.7 MeV release of nuclear energy. This reaction allows an estimate of the copious solar neutrino flux on Earth, assuming that the solar nuclear energy generation equals the Sun's luminosity and does not vary over time periods short in comparison to the nuclear time scale,

$$
\Phi_{\nu \odot}=\frac{2 L_{\odot}}{Q-2 E_{\nu}} \frac{1}{4 \pi(A U)^{2}} \approx 6.5 \times 10^{10} \nu_{\odot} \mathrm{cm}^{-2} s^{-1}
$$

where $L_{\odot}=3.86 \times 10^{33} \mathrm{ergs}^{-1}$ is the Suns's luminosity, $A U=1.5 \times 10^{13} \mathrm{~cm}$ is its average distance from Earth, and $E_{\nu} \approx 0.26 \mathrm{MeV}$ is the average energy of the produced neutrinos. The net reaction assumes that baryon number, charge flavour, and energy are conserved quantities. In the following it will be assumed that the reported results of the four neutrino experiments are correct. 


\section{Fundamental Physical Constants and Solar Structure: Differential Equations}

There are two distinct types of basic equations in physics: dynamical equations exhibiting the reversible (Newtonian) time and transport equations reflecting the irreversible (Boltzmann-Gibbsian) time. The Sun contains a large number of hydrogen atoms, and evolves as a main-sequence star in nuclear time scale, an extremely long time in comparison to thermal diffusion time (Helmholtz-Kelvin time scale) and to its fundamental pulsation mode. Accordingly, the time evolution of the Sun is managed by the change of its chemical composition governed by a system of coupled nonlinear kinetic equations which can be solved separately from the system of coupled partial differential equations of solar structure, boundary conditions, and the constraint that the model luminosity at the present epoch must be equal to the observed solar luminosity.

The four basic differential equations required to calculate the internal structure of the Sun are equations which represent respectively the distribution of mass within the Sun, the balance of gravity and pressure giving hydrostatic equilibrium (involving Newton's gravitational constant G), the outward flow of energy driven by the temperature gradient inside the Sun (involving the velocity of light c and Stefan's constant a), and the equation of nuclear energy generation within the Sun which continually replenishes that radiated away (involving Planck's quantum of action $\hbar$ ). In order to solve these differential equations, it is necessary to specify the equation of state (involving Boltzmann's constant k), the opacity of the solar material and the nuclear energy generation rates. Boundary conditions have to be satisfied at the surface and at the centre of the Sun. From a more general point of view with regard to the calculation of the internal structure of the Sun it is only necessary to make an assumption about the distribution of the energy sources within the Sun, not necessarily knowing what produces the energy. Due to this fact, calculations of the internal structure of solar-type stars made already considerable progress before the production of energy by nuclear synthesis was understood in any detail (Eddington 1928, cp. Chandrasekhar 1984). The differential equations for the internal structure of the Sun contain fundamental constants of physics that make it possible to gain qualitative insight into the solutions of the system of differential equations by 
simple dimensional analysis. In quantum field theory the Sommerfeld finestructure constant $\alpha_{e l}$ plays the role of a dimensionless coupling constant for the Coulomb force:

$$
\alpha_{e l}^{-1}=\frac{\hbar c}{e^{2}} \approx 137
$$

Equivalent to this quantity one can define the gravitational fine-structure constant $\alpha_{g}$,

$$
\alpha_{g}^{-1}=\frac{\hbar c}{G m_{p}^{2}} \approx 10^{38},
$$

where $m_{p}$ is the mass of the proton. This quantity measures the smallness of the gravitational force between two protons, similar to $\alpha_{e l}$ which measures the smallness of the Coulomb force between two electrons. In terms of stability of matter, $\alpha_{e l}^{-1}$ gives the maximum positive charge of the central nucleus that will allow a stable electron-orbit around it. It can be further shown that the combination of the fundamental constants in $\alpha_{e l}^{-1}$ and $\alpha_{g}^{-1}$ are playing an important role in the evolution and structure of the Sun as expected from the differential equations (Salpeter 1966). Electromagnetic and gravitational interactions are long range interactions, where the unit of electromagnetic interaction is e and the unit of gravitational interaction is $m_{p}\left(m_{p}=1837 m_{e}\right)$. The fourth fundamental constant of physics, Boltzmann's $\mathrm{k}$, is an exceptional case since temperature can be defined in terms of energy (Gamow 1970). However, if the equation of state for solar material followed exclusively the perfect gas law, one would not have any preferred units for density and temperature. This leads necessarily to the consideration of radiation pressure (involving Planck's constant $\hbar$ ) and electron degeneracy (involving $\hbar$ and the electron mass $m_{e}$ ) for the internal structure of the Sun.

\section{The Sun is Massive Because Gravity is Weak: Virial Theorem}

The Sun is a system of $\mathrm{N}$ nucleons with a mean separation of the order of magnitude d,

$$
M_{\odot} \approx N m_{p}, R_{\odot} \approx N^{1 / 3} d,
$$

where $M_{\odot}$ and $R_{\odot}$ denote the mass and radius of the Sun, respectively. Observationally the Sun ought to be considered close to hydrostatic equi- 
librium while from the theoretical point of view the Sun is considered to be in complete hydrostatic equilibrium. The virial theorem implies that the gravitational binding energy of the Sun must be of the order of its internal energy,

$$
3 \int d V P=\frac{G M_{\odot}^{2}}{R_{\odot}}=-\Omega_{g}=\frac{G m_{p}^{2} N^{5 / 3}}{d},
$$

where $\mathrm{P}$ is the total pressure in volume element $\mathrm{dV}$ and the right-hand side of the equation is the total gravitational potential energy of the Sun. The total thermal energy content of the Sun is the kinetic energy per particle times its number,

$$
E_{t h} \simeq N k T \text {. }
$$

Two forces balance to keep the Sun in hydrostatic equilibrium: the gravitational force directed inward and the gas and radiation pressure force directed outward. The total radiation energy is the product of the volume of the Sun and $a T^{4}$ :

$$
E_{r} \simeq N d^{3} a T^{4}
$$

where a is Stefan's constant, $a=\frac{\pi^{2}}{15} \frac{k^{4}}{c^{3} \hbar^{3}}$, by virtue of Planck's law. According to Heisenberg's uncertainty principle, the Fermi momentum of free electrons is $p_{F} \approx \hbar / d$, where $\mathrm{d}$ is the average separation of electrons. Provided the electrons are nonrelativistic, their Fermi energy is $E_{F}=p^{2} / 2 m_{e}$ and one obtains for the electron degeneracy energy,

$$
E_{d} \simeq N \frac{p^{2}}{2 m_{e}}=N \frac{\hbar^{2}}{2 m_{e} d^{2}}
$$

Thus, the virial theorem implies that

$$
N k T+N d^{3} a T^{4}+N \frac{\hbar^{2}}{2 m_{e} d^{2}} \approx N^{5 / 3} \frac{G m_{p}^{2}}{d} .
$$

For a star of solar mass with a mean molecular weight equal to 1 , the radiation pressure at the center cannot exceed a few percent of the total pressure and can be neglected. Similarly, electron degeneracy does not contribute to the total pressure under solar conditions. Hence,

$$
k T \simeq N^{2 / 3} \frac{G m_{p}^{2}}{d}=N^{2 / 3} \alpha_{g} \frac{\hbar c}{d}=\left(\frac{N}{N_{0}}\right)^{2 / 3} \frac{\hbar c}{d},
$$


with $N_{0}=\alpha_{g}^{-3 / 2}$. The Sun is massive because gravity is weak. This simple relationship between temperature and number of nucleons also confirms that the most important fact concerning a star is its mass. More detailed calculation shows that there exists an upper limit for the mass of a stable star of $\sim 100 N_{0} m_{p}$, otherwise radiation would dominate and lead to the disruption of it. Likewise a lower limit for a star's mass can be derived, $\sim 0.1 N_{0} m_{p}$, to account for the temperature needed to ignite nuclear fuel to form a selfsupported shining star, i.e., to burn hydrogen (Weisskopf 1975, Carr and Rees 1979, Dyson 1979).

The internal structure of a configuration $M \simeq 100 N_{0} m_{p}$ is dominated by radiation pressure, while for $M \geq 0.1 N_{0} m_{p}$ it is held together by electron degeneracy.

\section{Gravitationally Stabilized Solar Fusion Re- actor: Adjustment Factor}

The basic condition for thermonuclear reactions between charged particles is that their thermal energy must be large enough to penetrate the Coulomb repulsion between them. Nuclear reactions are collision phenomena characterized by cross sections. The cross section $\sigma$ of a reaction is defined as the probability that the reaction will occur if the incident flux consists of one particle and the target contains only one nucleus per unit area. The microscopic nature of the particles requires the quantum mechanical treatment of the collision problem. The number of reactions is directly proportional to the number density of the incident flux and the number density of the target. In the case of the nuclear fusion plasma within the Sun, thermal equilibrium is commonly assumed for the ensemble of nuclei. The distribution of the relative velocities among the nuclei is Maxwell-Boltzmannian. The thermonuclear reaction rate is given by

$$
r_{12}=n_{1} n_{2}<\sigma v>_{12}
$$

where $n_{1}$ and $n_{2}$ denote the number densities of particles of type 1 and 2 , respectively, and $\langle\sigma v\rangle_{12}$ is the reaction probability in the unit volume per unit time. This definition of the reaction rate reveals immediately that the quantity

$$
\tau_{12}=\left[n_{2}<\sigma v>_{12}\right]^{-1},
$$


has the dimension of time and can be considered to be the lifetime of particle 2 against reaction with particle 1 . A suitable representation of the nuclear cross section contains two factors: A geometrical factor to which quantum mechanical interaction between two particles is always proportional, $\lambda^{2} \sim$ $\left(\mu v^{2}\right)^{-1}$ (where $\lambda$ is the reduced de Broglie wave length, and $\mu$ is the reduced mass) and the probability for two particles of charge $Z_{1} e$ and $Z_{2} e$ to penetrate their electrostatic repulsion:

$$
\sigma(v)=\frac{2 S}{\mu v^{2}} \exp \left\{-2 \pi \frac{Z_{1} Z_{2} e^{2}}{\hbar v}\right\} .
$$

The constant $\mathrm{S}$ is called astrophysical cross section factor and absorbs the intrinsical nuclear parts of the probability for the occurrence of a nuclear reaction. Then, the reaction probability is defined as the product of the cross section $\sigma$ and the relative velocity $v$, averaged over the Maxwell-Boltzmann distribution of relative velocities of the reacting particles,

$$
f(v) d v=\left(\frac{\mu}{2 k T}\right)^{3 / 2} \exp \left\{-\frac{\mu v^{2}}{2 k T}\right\} 4 \pi v^{2} d v .
$$

To investigate the competition between the exponential factors contained in the Maxwell-Boltzmann distribution function and the Gamov penetration factor the following order of magnitude estimation is pursued. For the number density of the particle gas we use the mean density of the Sun with mass $M_{\odot}$ and radius $R_{\odot}$ normalized to the mass of the proton, $m_{p}$,

$$
n_{2}=\frac{M_{\odot}}{R_{\odot}^{3}} \frac{1}{m_{p}} .
$$

The velocity of the nuclei is assumed to be the root-mean-square velocity of the Maxwell-Boltzmann distribution,

$$
v_{12}=\left(\frac{4 k T}{m_{p}}\right)^{1 / 2} .
$$

The nuclear energy generated in the Sun, which is lost by radiation, can be estimated in writing

$$
E_{n u c} \approx X \Delta m M_{\odot} c^{2}
$$


where $\mathrm{X}$ is the fraction of mass the Sun can use for nuclear energy generation, $\Delta m M_{\odot} c^{2}$ is the fraction of mass of the Sun really converted into radiation energy. Thus, the nuclear lifetime of the Sun is of the order

$$
\tau^{-1} \approx \frac{L_{\odot}}{E_{\text {nuc }}} \approx \frac{L_{\odot}}{X \Delta m M_{\odot} c^{2}}
$$

For the lifetime of particle 2 one has

$$
\frac{1}{\tau_{12}} \approx \frac{L_{\odot}}{E_{\text {nuc }}} \approx n_{2} \sigma_{12} v_{12}
$$

Thus,

$$
\frac{L_{\odot}}{X \Delta m M_{\odot} c^{2}} \approx \frac{M_{\odot}}{R_{\odot}^{3} m_{p}} \frac{2 S}{m_{p}^{1 / 2}(k T)^{1 / 2}} \exp \left\{-\frac{2 \pi e^{2}}{\hbar}\left(\frac{m_{p}}{4 k T}\right)^{1 / 2}\right\}
$$

and isolating the exponential term in this expression by setting it equal to unity and than taking the logarithm, one gets

$$
2 \pi \alpha_{e l}\left(\frac{m_{p} c^{2}}{4 k T}\right)^{1 / 2} \approx \ln \left\{\frac{2 M_{\odot}^{2} X \Delta m S c^{2}}{L_{\odot} R_{\odot}^{3} m_{p}^{3 / 2}(k T)^{1 / 2}}\right\} .
$$

The numerical value of the logarithmic term on the right-hand-side in this equation is relatively insensitive to the values inserted for the various quantities in the brackets. Using solar values for the quantities, $M_{\odot} \approx 2 \times$ $10^{33} \mathrm{~g}, L_{\odot} \approx 3.86 \times 10^{33} \mathrm{ergs}^{-1}, R_{\odot} \approx 7 \times 10^{10} \mathrm{~cm}, T_{c \odot} \approx 10^{7} \mathrm{~K}, \mathrm{X}=0.1, \Delta \mathrm{m}=$ $0.007, S_{p p}=4 \times 10^{-22} \mathrm{keV}$ barn, one obtains for the logarithmic term a numerical value of about 10 . Then one obtains

$$
k T=\left(\frac{\left(2 \pi \alpha_{e l}\right)^{2}}{2^{2} 10^{2}}\right) m_{p} c^{2} \approx 5 k e V .
$$

This is the central temperature of the stationarily thermonuclear burning Sun. Actual central temperatures are about a factor 5 smaller or larger than this value due to the important fact that the majority of nuclear reactions occures in the high-energy tail of the Maxwell-Boltzmann distribution function. The Sun has to adjust this temperature through the competition between the distribution function of relative energies of the particles and the penetration factor of the reacting particles. 


\section{Numerical Simulation of the Five-Point Mov- ing Average of the Argon-Production Rate of the Chlorine Solar Neutrino Experiment : Variations Over Time}

The solar neutrino flux has been inferred from the neutrino capture rate in the chlorine neutrino experiment, measured over the past two decades in 86 separate runs (1970.8-1990.0). The chlorine detector with its threshold of 0.8 $\mathrm{MeV}$ is sensitive to the high energy, low flux part of the neutrino spectrum of the Sun, principally the neutrinos from the ${ }^{7} B e$ and ${ }^{8} B$ reactions. In the following we are not concerned with the discrepancy between the average rate and the predicted rate, but with a possible time dependence of the measured argon-production rate.

Fig.1. Shows the relative importance of the different branches of the proton-proton chain: PPI, PPII, PPIII, producing respectively 26.23 MeV, 25.62 MeV, and 19.29 MeV. The differences in the energy production are due to the energy loss carried off by the neutrinos. The relative importance of the different branches depends on the nuclear reaction rates and on the temperature and density structure inside the Sun. The lifetime of the first particle in each reaction is indicated. 
The proton-proton chain begins by fusion of two protons (Figure 1). This reaction produces the great majority of solar neutrinos $\left(\Phi_{\nu}(p p) \approx\right.$ $\left.10^{10} \nu \mathrm{cm}^{-2} s^{-1}, \Phi_{\nu}(p p) \sim T_{c}^{-1.2}\right)$, in which a proton decays into a neutron in the immediate vicinity of another proton; the two particles form a heavy variety of hydrogen known as deuterium, along with a positron and a neutrino. The deuterium nucleus produced by the pp-reaction fuses with another proton to form ${ }^{3} \mathrm{He}$ and a gamma ray. Most often, $86 \%$ of the time, the PPIbranch is completed when two ${ }^{3} \mathrm{He}$ nuclei fuse to form an alpha particle and two protons, which return to the beginning of the cycle. Approximately $14 \%$ of the time, however, ${ }^{3} \mathrm{He}$ instead fuses with an alpha particle, producing ${ }^{7} \mathrm{Be}$ and a gamma ray; the ${ }^{7} \mathrm{Be}$ then captures an electron, transmutes into ${ }^{7} \mathrm{Li}$ and emits a neutrino $\left(\Phi_{\nu}\left({ }^{7} \mathrm{Be}\right) \approx 10^{1} 0 \nu \mathrm{cm}^{-2} \mathrm{~s}^{-1}, \Phi_{\nu}\left({ }^{7} \mathrm{Be}\right) \sim T_{c}^{8}\right)$. ${ }^{7} B e$ fuses with a proton to produce two alpha particles and thus terminates the PPII-branch. In rare cases, about $0.02 \%$ of the time, ${ }^{7} B e$ fuses with a proton to produce radioactive ${ }^{8} B$, which beta decays into unstable ${ }^{8} B e$ and ultimately decays into two alpha particles, a positron and an energetic neutrino $\left(\Phi_{\nu}\left({ }^{8} B\right) \approx 10^{7} \nu \mathrm{cm}^{-2} s^{-1}, \Phi_{\nu}\left({ }^{8} B\right) \sim T_{c}^{18}\right)$. This reaction terminates the PPIII-branch and is extremely sensitive to the actual central temperature of the Sun.

Fig.2. Spatial distribution of neutrino sources in the gravitationally stabilized solar fusion reactor. The hep reaction occurs only rarely and does not influence the rates of the other reactions. 
Because the beta decay of ${ }^{8} B$ follows the fusion of ${ }^{7} B e$ and a proton, all nonstandard solar models predict more reduction of the ${ }^{8} B$ neutrino flux than the ${ }^{7} B e$ neutrino flux. Any reduction of the ${ }^{7} B e$ production rate affects ultimately both the ${ }^{8} B$ and ${ }^{7} B e$ neutrino flux equally. This fact seems to make cooler Sun models incompatible with the experimental data as the higher Kamiokande observed rate relative to the Homestake rate cannot be explained because cooler Sun models reduce the expected ${ }^{8} B$ flux more than the ${ }^{7} B$ e flux. Unless there is an independent mechanism to suppress only the ${ }^{7} B e$ neutrino emission, all nonstandard solar models are in contradiction to the solar neutrino data collected in the Kamiokande and Homestake experiments. The only astrophysical explanation of this phenomenon would be a greater reduction of temperature in the region of the energy production (i.e. in the vicinity of $0.1 R_{\odot}$ ) than just the central part where ${ }^{8} B$ neutrinos are produced (cp. Figure 2).

Fig.3. Five-point moving average of the argon-production rate versus time (in years) in the chlorine solar neutrino experiment for 86 runs in the time period 1970.8 to 1990.0. 
Fig.3 shows a five-point moving average of the argon-production rate, removing high frequency noise from the actual time series collected in the chlorine solar neutrino experiment (Haubold and Beer 1992). One notes in the five-point moving average that in the periods 1977 to 1980 and 1987 to 1990 a suppression of the argon-production rate occurs. The overall shape of the five-point moving average suggests that there are two distinctive epochs spanning the time periods 1970 to 1980 and 1980 to 1990, respectively. Each epoch shows a shock-like rise and after that a rapid decline of the argonproduction rate. The following simple mathematical model is able to simulate just this behaviour of the solar neutrino flux based on the lifetimes of concerned nuclear reactions of the PPIII-branch. For the time dependence of each of the relevant individual reactions in the PPIII-branch, the production rates for argon are assumed to follow

$$
y=k t
$$

where $y$ denotes the argon production rate and $t$ is the time. Then it is assumed that after a certain period the argon-production rate decreases to zero thus forming a triangle. This formulation is repeated over time. This type of simulation is motivated by a growth-decay mechanism discussed in Mathai (1993). Instead of a linear growth and decay a nonlinear growth and decay resulting in a bell-shaped function is also considered. That is,

$$
y=z r \sum_{j=0}^{v} \exp \left\{-\frac{(t-[r+x+2 j r])^{2}}{2(b)^{2}}\right\},
$$

where $z=k=\sqrt{3}$ is the slope of an equilateral triangle, $b=\sigma$ the confidence level, $x$ denotes one set of triangles delayed by $x$ units, $v$ is the number of respective triangles, and $r$ is the half of the triangle base. The two epochs in Fig.3 span a time period of approximately 10 years each which will be divided into 40 time units of 2.5 months each. The following table contains the parameters chosen for the three relevant nuclear reactions in the PPIIIbranch: For the simulation of the shape of the two epochs in Fig. 3 based on the parameters for the three nuclear reactions of PPIII-branch contained in Table 1 one has,

$$
y=z\left\{q \sum_{i=0}^{u} \exp \left\{-\frac{(t-[q+2 i q])^{2}}{2(a)^{2}}\right\}+\right.
$$




\begin{tabular}{|l|l|l|c|}
\hline $\begin{array}{l}\text { nuclear reaction } \\
\text { of PPIII-branch }\end{array}$ & lifetime & $\begin{array}{l}\text { number of triangles/ } \\
\text { half of the triangle } \\
\text { base }\end{array}$ & confidence level \\
\hline${ }^{7} \mathrm{Be}\left(e^{-}, \nu\right)^{7} \mathrm{Li}$ & $10^{-1} \mathrm{yr}$ & 40 months $/ 20$ & 6.7 \\
\hline${ }^{7} \mathrm{Li}(p, \alpha)^{4} \mathrm{He}$ & $10^{-5} \mathrm{yr}$ & 8 month $/ 4$ & 1.3 \\
\hline${ }^{8} \mathrm{~B}\left(e^{+}, \nu\right)^{8} \mathrm{Be} e^{*}$ & $10^{-8} \mathrm{yr}$ & 5 month $/ 2.5$ & 0.8 \\
\hline
\end{tabular}

Table 1:

$$
\begin{aligned}
& r \sum_{j=0}^{v} \exp \left\{-\frac{(t-[r+x+2 j r])^{2}}{2(b)^{2}}\right\}+ \\
& \left.s \sum_{k=0}^{w} \exp \left\{-\frac{(t-[s+2 k s])^{2}}{2(c)^{2}}\right\}\right\} .
\end{aligned}
$$

The result of the numerical computation for $y(t ; a, b, c ; z ; q, r, s ; x ; u, v, w)=y(t ; 0.8,1.3,6.7 ; 1.7 ; 2.5,4,20 ; 3 ; 7 ; 4,0)$ is shown in Fig. 4.

Fig.4. Numerical simulation of one 10-year epoch revealed by the time dependence 
of the five-point moving average of the argon production rate. The simulation is based upon the lifetimes of nuclear reactions of the PPIII-branch, producing the ${ }^{7} B e$ and ${ }^{8} B$ neutrinos.

\section{REFERENCES}

O.L. Anosov et al., Nucl. Phys. Suppl. B31, 111 (1993).

P. Anselmann et al., Phys. Rev. Lett., submitted (1994).

J.N. Bahcall and M. Pinsonneault, Rev. Mod. Phys. ㄸ4, 885 (1992).

B.J. Carr and M.J. Rees, Nature 278, 605 (1979).

S. Chandrasekhar, Rev. Mod. Phys. ㅌ6, 137 (1984).

R. Davis Jr., in Frontiers of Neutrino Astrophysics (Eds. Y. Suzuki and K. Nakamura), Universal Academy Press, Inc., Tokyo, 1993, pp. 47.

F.J. Dyson, Rev. Mod. Phys. 1ㅗ, 447 (1979).

G. Gamow, The Three Kings of Physics. In Physics, Logic, and History (Eds. W. Yourgrau and A.D. Breck), Plenum Press, New York 1970, pp.203.

H.J. Haubold and J. Beer, Solar Activity Cycles Revealed by Time Series Analysis of Argon-37, Sunspot-Number, and Beryllium-10 Records. In Solar-Terrestrial Variability and Global Change (Eds. W. Schroeder and J.P. Legrand), Proceedings of the IUGG/IAGA General Assembly, Vienna 1991, pp.11.

A.M. Mathai, Can. J. Statistics 21, 277 (1993).

K. Nakamura, Nucl. Phys. Suppl. B31, 105 (1993).

E.E. Salpeter, Dimensionless Ratios and Stellar Structure.

In Perspectives in Modern Physics (Ed. R. Marshak), John Wiley and Sons, New York, 1966, pp. 463.

V.F. Weisskopf, Science 187, 605 (1975). 
This figure "fig1-1.png" is available in "png" format from: http://arxiv.org/ps/astro-ph/9405040v1 
This figure "fig1-2.png" is available in "png" format from: http://arxiv.org/ps/astro-ph/9405040v1 